IMTC 2007 - IEEE Instrumentation and Measurement

Technology Conference

Warsaw, Poland, May 1-3, 2007

\title{
Temperature Dependence of Fixed Pattern Noise in Logarithmic CMOS Image Sensors
}

\author{
Dileepan Joseph ${ }^{1}$ and Steve Collins ${ }^{2}$ \\ ${ }^{1}$ Department of Electrical and Computer Engineering, University of Alberta \\ 9107 116th St NW, Edmonton, AB, T6G 2V4, Canada \\ Phone: +1 780 4929164, Fax: +1 7804921811 \\ Email: dil.joseph@ualberta.ca \\ ${ }^{2}$ Department of Engineering Science, University of Oxford \\ Parks Road, Oxford, OX1 3PJ, United Kingdom \\ Phone: +44 1865 273151, Fax: +44 1865273905 \\ Email: collins@ robots.ox.ac.uk
}

\begin{abstract}
This paper presents a model that explains the contribution of temperature to the fixed pattern noise (FPN) in a logarithmic CMOS image sensor. Based on this model, a simpler model is proposed to facilitate the calibration and correction of FPN. To avoid nonlinear optimization, the variation of photodiode leakage current from one pixel to another is neglected. The simplified model uses the dark response of pixels, which depends only on temperature, to help predict FPN in the light response, which depends on temperature and illuminance. Calibration requires images of a uniform stimulus taken at different temperatures and illuminances, which need not be measured. After calibration, FPN is corrected in an arbitrary image using a dark image at the same temperature, which is taken infrequently. Through simulation, using mismatch data from a real CMOS process, an improvement is shown in the residual error per image after calibration, when the proposed method is compared to an established method that does not account for temperature dependence.
\end{abstract}

\section{INTRODUCTION}

Electronic circuits in consumer, industrial and military applications are required to operate in diverse and changing temperatures [1]. Unlike the human eye, image sensors usually do not exist in a homeostatic environment. Thus, due to semiconductor physics, the response of a pixel to illumination may change with temperature. Moreover, a pixel-to-pixel variation of device parameters related to temperature and/or illuminance sensitivity will lead to a fixed pattern noise (FPN) in acquired images. Whereas previous work by Joseph and Collins [2] considered FPN in logarithmic CMOS image sensors at one temperature, this paper considers the dependence of FPN on temperature itself.

Marshall and Collins [3] and Loose et al [4] noted that temperature would affect threshold voltage variation in logarithmic CMOS image sensors. A uniform temperature dependence would be less problematic than nonuniformity in the temperature dependence of pixel responses. However, the dependence was never characterized. Instead, Marshall and Collins suggested a digital method to correct FPN that considered both temperature and illumination dependence. They advocated using an autofocus system to defocus a scene to obtain a calibration image that may then be subtracted from the focused image of the scene. This approach required frequent mechanical operation and introduced spatial high pass filtering to the image, proving unsuitable when rendering images for human observers.

In the study of linear CCD and CMOS image sensors, it is well known that the response of pixels with the aperture of the camera closed, called the dark response, is a function of temperature [1]. In reality, this dark response also bears upon the response of the pixels with the aperture open (i.e. to a focused image), called the light response. As the dark response is only affected by temperature and not illuminance, this paper shows it may be used to calibrate and correct temperature-dependent FPN in the light response. Illumination-dependent FPN, which is also present, may be compensated using a method similar to one that has been previously established [2].

Section II models the response of a logarithmic pixel to temperature and illuminance. Section III describes calibration of a sensor using images of a uniform scene taken at different temperatures and with different illuminances. As calibration may be a costly process, emphasis is placed on eliminating temperature and illuminance measurement, reducing the complexity of the model and reducing the number of parameters requiring estimation. Finally, Section IV evaluates the simplified model and calibration method through simulation.

\section{MODELLING}

In a typical logarithmic CMOS image sensor, the digital response $y$ of a pixel depends on the light stimulus $x$ falling on 
the pixel according to the model [2]

$$
y=a+b \ln (c+x)+\epsilon,
$$

with an offset

$$
\begin{aligned}
a= & F_{A D C}+G_{A D C}\left(V_{D D}-V_{o n}^{\mathrm{T} 1}-V_{T}^{\mathrm{T} 2}-V_{T}^{\mathrm{T} 5}\right. \\
& -\sqrt{\frac{K^{\mathrm{T} 4}}{K^{\mathrm{T} 2}}}\left(V_{G S}^{\mathrm{T} 4}-V_{T}^{\mathrm{T} 4}\right)-\sqrt{\frac{K^{\mathrm{T} 7}}{K^{\mathrm{T} 5}}}\left(V_{G S}^{\mathrm{T} 7}-V_{T}^{\mathrm{T} 7}\right) \\
& \left.+\frac{n^{\mathrm{T} 1} k T}{q} \ln \frac{I_{o n}^{\mathrm{T} 1}}{G_{A} G_{L} G_{Q} A}\right),
\end{aligned}
$$

a gain

$$
b=-G_{A D C} \frac{n^{\mathrm{T} 1} k T}{q},
$$

and a bias

$$
c=\frac{I_{S}}{G_{A} G_{L} G_{Q} A} .
$$

The error $\epsilon$, which is made up of quantization, temporal and approximation noise, is assumed to be independent of temperature $T$ and illuminance $x$. Superscripts identify the ownership of parameters by numbered transistors in Figure 1 of Reference [2], the source of (1)-(4). For example, $V_{G S}^{\mathrm{T} 4}$ and $V_{G S}^{\mathrm{T} 7}$ are the column and output source follower biases respectively. Furthermore, $F_{A D C}$ and $G_{A D C}$ are the offset and gain of the ADC, $V_{D D}$ is the supply voltage and $G_{A}, G_{L}$ and $G_{Q}$ are gains due to the aperture, lens and quantum efficiency. Other circuit parameters are described below.

The offset $a$ in (2), an abstract parameter that incorporates a number of physical parameters, is affected by temperature in several ways. To begin with, threshold voltages $V_{T}$ have a linear dependence on temperature and current gains $K$ depend on temperature by a power law, i.e.

$$
\begin{aligned}
V_{T} & =V_{T 0}-T C V\left(T-T_{r e f}\right), \\
K & =K_{0}\left(\frac{T}{T_{r e f}}\right)^{B E X} .
\end{aligned}
$$

These equations come from the HSPICE Level 28 model [5] as simpler models do not consider the temperature dependence of $V_{T}$ or $K . T_{r e f}$ is a reference temperature, measured in Kelvins as with $T . V_{T 0}$ and $K_{0}$ are the threshold voltage and current gain at that temperature. The multiplier $T C V$ and exponent $B E X$ define how quickly the threshold voltage and current gain change with temperature. For simplicity, it is assumed that $B E X$ does not vary from transistor to transistor.

The parameter $V_{o n}$ in (2), taken from the Level 3 model [5], signifies the gate-source voltage at which a transistor changes from the weak to the strong inversion region of operation. This threshold depends linearly on temperature, i.e.

$$
V_{o n}=V_{T}+\frac{n k T}{q} \text {. }
$$

The parameter $I_{o n}$ in (2) is the drain-source current at the gatesource voltage $V_{o n}$. Its dependence on temperature may be found using the Level 1 model [5] of current in the saturation region (ignoring the finite output resistance), i.e.

$$
I_{o n}=K\left(\frac{n k T}{q}\right)^{2} .
$$

Assuming an ideal ADC, the gain $b$ depends on temperature in only one way, as shown in (3). The relationship comes from the Level 3 model [5], wherein the slope of the subthreshold response $\left(V_{D S}\right.$ versus the logarithm of $\left.I_{D S}\right)$ of a diodeconnected transistor (where $V_{G S}$ equals $V_{D S}$ ) is a multiple of temperature, i.e.

$$
V_{D S}=\frac{n k T}{q} \ln \frac{I_{D S}}{I_{o n}}+V_{o n} .
$$

In this equation, $n$ is an ideality factor, $k$ is the Boltzmann constant and $q$ is the electron charge.

Neglecting any dependence of the aperture, lens and quantum efficiency gains on temperature, the bias $c$ in (4) depends on temperature in only one way also. In the simplest case [5], the reverse bias saturation current of the photodiode is an exponential function of temperature, i.e.

$$
I_{S}=I_{S 0} e^{T / T_{e}} .
$$

The parameter $T_{e}$ equals about $\frac{7}{\ln 2} \mathrm{~K}$ since $I_{S}$ doubles approximately every $7 \mathrm{~K}[1]$. Although $I_{S 0}$ may vary from device to device, variation of $T_{e}$ is neglected for simplicity.

Factoring the above temperature dependences of physical parameters out of (2)-(4), the response $y$ of a logarithmic pixel to temperature $T$ and illuminance $x$ is given by

$$
y=a_{1}+a_{2} T+a_{3} T \ln T+b_{1} T \ln \left(c_{1} e^{T / T_{e}}+x\right)+\epsilon,
$$

with offsets $a_{1}, a_{2}$ and $a_{3}$, a gain $b_{1}$ and a bias $c_{1}$. Using (1)(11), formulas for these abstract parameters may be derived [6]. A pixel-to-pixel variation of $a_{1}, a_{2}, a_{3}, b_{1}, c_{1}$ or any combination thereof would cause FPN in an image sensor. Unless only $a_{1}$ varied, any type of FPN will depend on temperature.

\section{CALIBRATION}

One way to correct FPN in an image sensor is to estimate the parameters that vary from pixel to pixel by calibration and to compensate accordingly for variation. Using the model in (1), which does not consider temperature dependence, three types of FPN have been examined in the literature [2]: single variation, where only $a$ varies; double variation, where $a$ and $b$ vary; and triple variation, where $a, b$ and $c$ vary. Given images of a uniform scene, taken at one temperature but at different illuminances, the single and double variation models may be calibrated by linear regression, permitting an analytic solution, 
and the triple variation model may be calibrated by nonlinear optimization, requring an iterative solution.

To calibrate a sensor having $N$ pixels over both temperature and illuminance, images are taken of a uniform scene at $L$ different temperatures $T_{h}$, where $1 \leq h \leq L$, and $M$ different nonzero illuminances $x_{i}$, where $1 \leq i \leq M$. These $L \times M$ images comprise the light response of the sensor. Images are also taken at the same temperatures but with zero illuminance, e.g. by closing the aperture of the camera. These $L$ images comprise the dark response of the sensor. At the $h^{\text {th }}$ temperature and the $i^{\text {th }}$ illuminance, the actual light and dark responses of the $j^{\text {th }}$ pixel, where $1 \leq j \leq N$, are denoted $y_{h i j}$ and $y_{h 0 j}$. Using (11), these responses are predicted by

$$
\begin{gathered}
\tilde{y}_{h i j}=a_{1 j}+a_{2 j} T_{h}+a_{3 j} T_{h} \ln T_{h}+b_{1 j} T_{h} l_{h i}, \\
\tilde{y}_{h 0 j}=a_{1 j}+a_{2 j} T_{h}+a_{3 j} T_{h} \ln T_{h}+b_{1 j} T_{h} l_{h 0},
\end{gathered}
$$

where

$$
\begin{aligned}
& l_{h i}=\ln \left(c_{1} e^{T_{h} / T_{e}}+x_{i}\right), \\
& l_{h 0}=\ln \left(c_{1} e^{T_{h} / T_{e}}\right) .
\end{aligned}
$$

The differences between the actual responses $y_{h i j}$ and $y_{h 0 j}$ and the predicted responses $\tilde{y}_{h i j}$ and $\tilde{y}_{h 0 j}$ are error terms $\epsilon_{h i j}$ and $\epsilon_{h 0 j}$, which are assumed to be independent from sample to sample and to follow a zero-mean Gaussian distribution.

Note that (12) and (13) imply a variation of three offset ( $a_{1}$, $a_{2}$ and $\left.a_{3}\right)$ and one gain $\left(b_{1}\right)$ parameter from pixel to pixel, representing $4 N$ variables. As bias variation has not been considered, there are two variables $c_{1}$ and $T_{e}$ in (14) and (15). While bias variation does exist in logarithmic imagers [2], it is not practical to account for it because calibration would require nonlinear optimization. Moreover, whereas temperatures $T_{h}$ and illuminances $x_{i}$ may be measured, calibration would be simpler if their values were not required.

Fortunately, there is a way to calibrate the image sensor while avoiding nonlinear optimization and measurement of $T_{h}$ and $x_{i}$. This method relies on two observations, namely that the averages, taken over all pixels, of the actual and predicted responses are interchangeable for either the light or the dark case, i.e.

$$
\begin{aligned}
\frac{1}{N} \sum_{j=1}^{N} y_{h i j} & \approx \frac{1}{N} \sum_{j=1}^{N} \tilde{y}_{h i j}, \\
\frac{1}{N} \sum_{j=1}^{N} y_{h 0 j} & \approx \frac{1}{N} \sum_{j=1}^{N} \tilde{y}_{h 0 j} .
\end{aligned}
$$

These approximations are good when there are many pixels because averaging reduces the variance of the error terms, i.e. the difference between actual and predicted responses.

Denoting the left sides of (16) and (17) by $\bar{y}_{h i}$ and $\bar{y}_{h 0}$, called the average light and dark responses, and substituting
(12) and (13) into the right sides gives

$$
\begin{aligned}
& \bar{y}_{h i} \approx \bar{a}_{1}+\bar{a}_{2} T_{h}+\bar{a}_{3} T_{h} \ln T_{h}+\bar{b}_{1} T_{h} l_{h i}, \\
& \bar{y}_{h 0} \approx \bar{a}_{1}+\bar{a}_{2} T_{h}+\bar{a}_{3} T_{h} \ln T_{h}+\bar{b}_{1} T_{h} l_{h 0},
\end{aligned}
$$

where $\bar{a}_{1}, \bar{a}_{2}$ and $\bar{a}_{3}$ are the average offsets, taken over all pixels, and $\bar{b}_{1}$ is the average gain. Examination of (19) and (15) shows that $\bar{y}_{h 0}$ is a function of $T_{h}$ with constants $\bar{a}_{1}, \bar{a}_{2}, \bar{a}_{3}, \bar{b}_{1}$, $\bar{c}_{1}$ and $T_{e}$. Thus, there is no need to measure temperature since the average dark response may be used in proxy.

To eliminate the measurement of $x_{i}$, which appears in (18) and (12) thanks to (14), one substitutes the left side of (18) into the right side of (12) to give

$$
\tilde{y}_{h i j} \approx a_{1 j}^{\prime}+a_{2 j}^{\prime} T_{h}+a_{3 j}^{\prime} T_{h} \ln T_{h}+b_{1 j}^{\prime} \bar{y}_{h i},
$$

with a normalized gain

$$
b_{1 j}^{\prime}=b_{1 j} / \bar{b}_{1},
$$

and normalized offsets

$$
a_{k j}^{\prime}=a_{k j}-b_{1 j}^{\prime} \bar{a}_{k},
$$

where $1 \leq k \leq 3$.

What remains is to eliminate $T_{h}$ in (20) using $\bar{y}_{h 0}$ in (19). The nonlinearities $T_{h} \ln T_{h}$ and $T_{h} l_{h 0}$, where $l_{h 0}$ is a function of $T_{h}$ by (15), make this impossible. However, assuming $\bar{y}_{h 0}$ approximates a linear function of $T_{h}$ over the temperature range of interest, $T_{h} \ln T_{h}$ and $T_{h} l_{h 0}$ may be linearized. Using Taylor expansion around an operating point $\bar{T}$, one finds

$$
\begin{aligned}
T_{h} \ln T_{h} & \approx T_{h}(1+\ln \bar{T})-\bar{T}, \\
T_{h} l_{h 0} & \approx T_{h}\left(\ln c_{1}+2 \bar{T} / T_{e}\right)-\bar{T}^{2} / T_{e} .
\end{aligned}
$$

With these linearizations, the left side of (19) may be substituted into the right side of (20) to give

$$
\tilde{y}_{h i j} \approx a_{1 j}^{\prime \prime}+a_{2 j}^{\prime \prime} \bar{y}_{h 0}+b_{1 j}^{\prime} \bar{y}_{h i},
$$

with linearized offsets

$$
a_{l j}^{\prime \prime}=\frac{1}{d_{11}} \sum_{k=1}^{3} d_{l k} a_{k j}^{\prime},
$$

where $1 \leq l \leq 2$ and where $d_{l k}$ are linearization coefficients. Using (20)-(26), formulas for $d_{l k}$ may be derived [6].

Equation (25), called the temperature proxy model, shows that the predicted light response of any pixel is approximately a linear function of the average dark and light responses of all pixels, given the same temperature and illuminance. There is no need to determine any of the linearization coefficients $d_{l k}$ in (26) if the linearized offsets $a_{l j}^{\prime \prime}$ and normalized gain $b_{1 j}^{\prime}$ in (25) are estimated directly. These unknowns are estimated for all pixels by minimizing the sum square error (SSE) between the actual and predicted light responses, i.e.

$$
\operatorname{SSE}\left(a_{1 j}^{\prime \prime}, a_{2 j}^{\prime \prime}, b_{1 j}^{\prime}\right)=\sum_{h=1}^{L} \sum_{i=1}^{M} \sum_{j=1}^{N}\left(y_{h i j}-\tilde{y}_{h i j}\right)^{2} .
$$


Using multilinear algebra [6], the estimated parameters are

$$
\hat{\mathbf{b}}_{j}=\left(\sum_{h=1}^{L} \sum_{i=1}^{M} \overline{\mathbf{y}}_{h i}^{\mathrm{T}} \overline{\mathbf{y}}_{h i}\right)^{-1}\left(\sum_{h=1}^{L} \sum_{i=1}^{M} \overline{\mathbf{y}}_{h i}^{\mathrm{T}} y_{h i j}\right),
$$

where

$$
\begin{aligned}
\hat{\mathbf{b}}_{j} & =\left(\begin{array}{lll}
\hat{a}_{1 j}^{\prime \prime} & \hat{a}_{2 j}^{\prime \prime} & \hat{b}_{1 j}^{\prime}
\end{array}\right)^{\mathrm{T}}, \\
\overline{\mathbf{y}}_{h i} & =\left(\begin{array}{lll}
1 & \bar{y}_{h 0} & \bar{y}_{h i}
\end{array}\right) .
\end{aligned}
$$

Note that in addition to the $3 N$ variables $\hat{a}_{1 j}^{\prime \prime}, \hat{a}_{2 j}^{\prime \prime}$ and $\hat{b}_{1 j}^{\prime}$ calculated from the actual light response $y_{h i j}$ according to (28), the $L M$ variables $\bar{y}_{h i}$ are also calculated from the same response according to the left side of (16). Thus, there are

$$
P=L M+3 N
$$

model parameters explicitly fitted to the acquired data. Using only (28)-(30), it is possible to prove that

$$
\frac{1}{N} \sum_{j=1}^{N}\left(\begin{array}{lll}
\hat{a}_{1 j}^{\prime \prime} & \hat{a}_{2 j}^{\prime \prime} & \hat{b}_{1 j}^{\prime}
\end{array}\right)=\left(\begin{array}{lll}
0 & 0 & 1
\end{array}\right),
$$

which means three parameters may be calculated without using the data. Thus, there are actually

$$
Q=L M+3 N-3
$$

parameters implicitly fitted to the data.

The term inside parentheses on the right side of (27) is merely the error term $\epsilon_{h i j}$. Thus, an estimate of the error variance may be found by dividing the minimized SSE by the corresponding degrees of freedom, i.e.

$$
\hat{\sigma}_{\epsilon}^{2}=\frac{S S E\left(\hat{a}_{1 j}^{\prime \prime}, \hat{a}_{2 j}^{\prime \prime}, \hat{b}_{1 j}^{\prime}\right)}{L M N-Q} .
$$

The denominator equals the number of responses $L M N$ minus the number of implicit parameters $Q$ fitted to those responses (using $P$ would be slightly incorrect).

In theory, the estimated variance of the error term has the same expected value even if the estimate were made using data from only one temperature and illuminance, i.e. using data from only one image. Hence, the SSE per image, a function of temperature and illuminance, is given by

$$
S S E_{h i}\left(a_{1 j}^{\prime \prime}, a_{2 j}^{\prime \prime}, b_{1 j}^{\prime}\right)=\sum_{j=1}^{N}\left(y_{h i j}-\tilde{y}_{h i j}\right)^{2},
$$

and the estimate of the error variance per image is given by

$$
\hat{\sigma}_{\epsilon_{h i}}^{2}=\frac{S S E_{h i}\left(\hat{a}_{1 j}^{\prime \prime}, \hat{a}_{2 j}^{\prime \prime}, \hat{b}_{1 j}^{\prime}\right)}{N-\frac{Q}{L M}} .
$$

For brevity, the square root of the estimated variances in (34) and (36), which represent estimated standard deviations, are called the residual error and the residual error per image. These statistics are useful in assessing the calibration.

The calibration was derived for images taken at all combinations of $L$ temperatures $T_{h}$ and $M$ illuminances $x_{i}$. In practice, the temperature of the image sensor is varied and, at each setpoint, the illuminance of the uniform scene is varied. But it may be difficult to ensure that the illuminance setpoints are the same from one temperature setpoint to another. In the worst case, $L M$ different illuminances, denoted $x_{h i}$, would occur. Fortunately, this change does not affect any of the above results. Although $l_{h i}$ in (14) must be rewritten as

$$
l_{h i}=\ln \left(c_{1} e^{T_{h} / T_{e}}+x_{h i}\right)
$$

none of the other equations need alteration.

Only a few formulas presented in this section require implementation. The left sides of (16) and (17) give $\bar{y}_{h i}$ and $\bar{y}_{h 0}$. Equation (28) gives $\hat{a}_{1 j}^{\prime \prime}, \hat{a}_{2 j}^{\prime \prime}$ and $\hat{b}_{1 j}^{\prime}$. Other formulas were presented to explain the method and provide a means for assessment. The calibration need only be done once. To correct FPN in an arbitrary image $y_{1 j}$ at any temperature, one requires a dark image $y_{0 j}$ at the same temperature, which may be taken infrequently with a closed aperture. A similar analysis to the above shows that the corrected image $y_{* j}$ is given by

$$
y_{* j}=\left(y_{1 j}-\hat{a}_{1 j}^{\prime \prime}-\hat{a}_{2 j}^{\prime \prime} \bar{y}_{0}\right) / \hat{b}_{1 j}^{\prime \prime},
$$

where $\bar{y}_{0}$ is the mean of the dark image.

As noted at the beginning of this section, bias variation has been neglected. To consider it, one must replace $l_{h i}$ and $l_{h 0}$ in (12) and (13) with $l_{h i j}$ and $l_{h 0 j}$, where

$$
\begin{aligned}
& l_{h i j}=\ln \left(c_{1 j} e^{T_{h} / T_{e}}+x_{h i}\right), \\
& l_{h 0 j}=\ln \left(c_{1 j} e^{T_{h} / T_{e}}\right) .
\end{aligned}
$$

With these changes, it is still possible to derive a calibration that eliminates measurement of both $T_{h}$ and $x_{h i}$. However, whether or not either of these measurements are eliminated, nonlinear optimization cannot be avoided.

Lastly, another method exists to calibrate and correct the FPN without measurement of $T_{h}$ or $x_{h i}$ and which does not require nonlinear optimization when bias variation is neglected. This method involves subtracting the dark from the light response and leads to an equation called the offset cancellation model [6]. However, the temperature proxy model proves superior. Therefore, the offset cancellation model has been omitted from this paper for brevity.

\section{SIMULATION}

A logarithmic CMOS image sensor [2] was simulated for a $0.35 \mu \mathrm{m} 3.3 \mathrm{~V}$ process from Austria Microsystems (AMS) using Cadence. Avoiding optical considerations, the stimulus $x$ 
was represented by an ideal current source, in parallel with the reverse-biased diode in the pixel. The ADC was not simulated and, therefore, what would be the ADC input voltage was taken as the response $y$. These changes affect neither the abstract model of Section II nor the calibration method of Section III.

Transistors and diodes were simulated using the BSIM3 Version 3 models [5] with parameters supplied by AMS [7]. Considering the design rules of the process [8], the nominal width of all transistors was set to $1 \mu \mathrm{m}$ and the nominal length was set to $0.6 \mu \mathrm{m}$. A model that describes the $\mathrm{p}-\mathrm{n}$ junction formed between n-type diffusion and p-type substrate was used to represent diodes. As these diodes simulated photodiodes in pixels, they were set to a $6.32 \mu \mathrm{m} \times 6.32 \mu \mathrm{m}$ size, which corresponds to a photosensitive square in a $10 \mu \mathrm{m} \times 10 \mu \mathrm{m}$ pixel with a $40 \%$ fill factor - the specifications of the HDRC VGA 2 logarithmic pixels built in a $0.35 \mu \mathrm{m} 3.3 \mathrm{~V}$ process by IMS Chips [9].

A simultaneous parametric, DC and Monte Carlo analysis was performed. The parametric analysis varied the temperature from 0 to $60^{\circ} \mathrm{C}$ in $5^{\circ} \mathrm{C}$ steps for a total of thirteen setpoints. The DC analysis varied the photocurrent in half-decade steps from $1 \mathrm{pA}$ to $1 \mu \mathrm{A}$ for a total also of thirteen setpoints. Thirdly, the Monte Carlo analysis repeated the simulation 100 times, each time with different device parameters for each transistor according to statistical distributions specified by AMS to simulate device mismatch. This approach simulates the response over temperature and photocurrent of 100 randomly selected pixels from a larger image sensor that suffers from FPN.

The simulated light response is denoted $y_{h i j}$, where $h$ indexes the temperature $T_{h}$, with $1 \leq h \leq 13, i$ indexes the photocurrent $x_{i}$, with $1 \leq i \leq 13$, and $j$ indexes the pixel, with $1 \leq j \leq 100$. In the literature on logarithmic imagers, noise levels may be quoted as a percentage of the change in response due to a one decade change in stimulus. Therefore, it is worth noting that the light response changed on average, over all temperatures and pixels, by $40 \mathrm{mV}$ per decade of photocurrent change. By setting the photocurrent to zero and carrying out the parametric and Monte Carlo analysis as before with the same random seed, the dark response of the pixels, denoted $y_{h 0 j}$, was simulated over the same temperatures and device parameters as before.

The light response at $30^{\circ} \mathrm{C}$ was used to calibrate the established double-variation model [2], which does not consider temperature dependence. The calibrated model was then used to estimate the light response at other temperatures. Figure 1 shows the residual error per image versus temperature and photocurrent for the calibration at $30^{\circ} \mathrm{C}$ and the extrapolation to other temperatures. A cross-section of this surface at any photocurrent is v-shaped, with a minimum at $30^{\circ} \mathrm{C}$, and a crosssection at any temperature is horizontal, not $\mathrm{w}$-shaped as reported in the literature. A $\mathrm{w}$-shape arises if the double variation model is used to calibrate pixels that actually obey the triple variation model, in which the photodiode leakage current varies from one pixel to another. AMS did not supply statistical

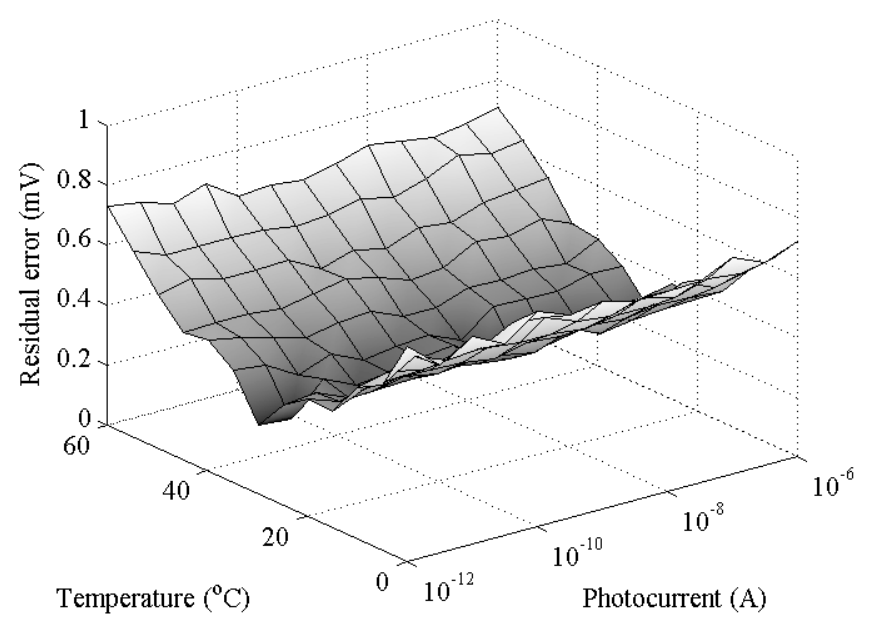

Fig. 1. The residual error per image versus temperature and photocurrent for calibration of the established double-variation model [2] to simulated data at $30^{\circ} \mathrm{C}$ with subsequent extrapolation to other temperatures.

distributions for parameters of the diode model so the leakage current did not vary in the mismatch simulation.

Consequently, at any one temperature, double variation is a perfect model of FPN in the simulated pixels but it is inaccurate away from the temperature of calibration. The degradation is graceful, which means the temperature dependence of FPN may be ignored if the temperature range is sufficiently small and the temperature of calibration falls in the middle. At $30^{\circ} \mathrm{C}$ alone, the residual error (over all photocurrents and pixels) was $0.29 \mathrm{mV}$ or $0.73 \%$ of a decade. Over the $0-60^{\circ} \mathrm{C}$ range, the residual error was $0.49 \mathrm{mV}$ or $1.2 \%$ of a decade.

Figure 2 shows that the average dark response of the simulated pixels may be accurately described as a linear proxy of temperature over the $0-60^{\circ} \mathrm{C}$ range. With this assumption confirmed, the light response was used to evaluate the temperature proxy model. After calibration, the residual error $\hat{\sigma}_{\epsilon}$, defined in (34), equalled $0.29 \mathrm{mV}$ or $0.73 \%$ of a decade. This result, obtained over the entire temperature range, is as good as the double variation result at $30^{\circ} \mathrm{C}$ alone and is better than the double variation result over the $0-60^{\circ} \mathrm{C}$ range. Figure 3 shows the residual error per image $\hat{\sigma}_{\epsilon_{h i}}$, defined in (36), versus temperature and photocurrent. The error is independent of both variables, unlike in Figure 1, meaning the temperature proxy model is suitable for extrapolation over wide ranges.

\section{CONCLUSION}

The response of a logarithmic pixel depends on temperature, as well as illuminance, because the threshold voltages, current gains, subthreshold slope, crossover current and leakage current of the circuit depend on temperature. Following semiconductor theory, a model of pixel response to tempera- 


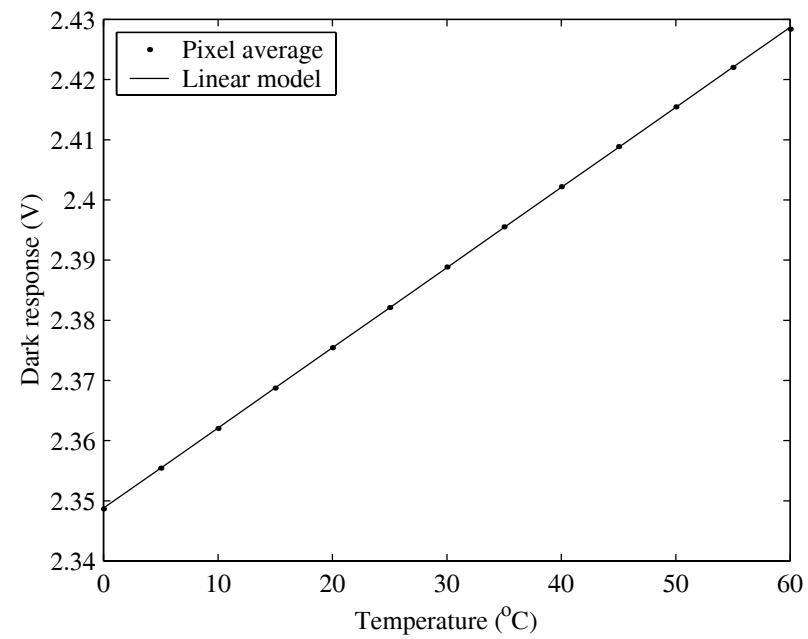

Fig. 2. The average dark response of 100 simulated pixels versus temperature. Regression analysis shows the relationship to be very linear.

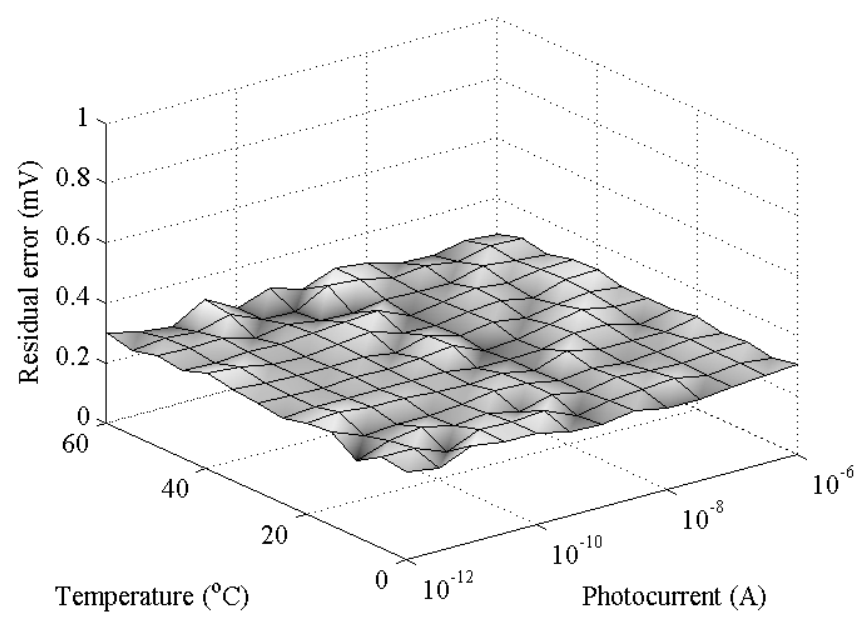

Fig. 3. The residual error per image versus temperature and photocurrent for calibration of the temperature proxy model to simulated data.

ture and illuminance has been derived, which incorporates offset, gain and bias parameters. A variation of any parameter or combination thereof from one pixel to another causes fixed pattern noise (FPN), which depends on temperature in all but one scenario.

Based upon the above model, a simpler temperature proxy model was proposed that is suitable for calibration and correction of the FPN. Calibration requires the light and dark responses of pixels to a uniform stimulus at several temperatures and illuminances. But it does not require measurement of any temperature or illuminance. If the average dark response of all pixels is a linear function of temperature then the light response of any pixel is a linear function of the average dark and light responses of all pixels, given the same temperature and illuminance. Correction of FPN in an arbitrary image follows readily. For the calibration and correction, bias variation has been neglected to avoid nonlinear optimization.

The above theory was validated through simulation of logarithmic pixels with mismatch data from a real process. Using the temperature proxy model, FPN may be calibrated to give a residual error that is independent of temperature, unlike with the previous work of Joseph and Collins [2], which employed a model that did not consider temperature. However, the degradation of the previous model is graceful, which means the temperature dependence of FPN may be safely ignored for a deviation on the order of $\pm 10^{\circ} \mathrm{C}$ with respect to the temperature of calibration. To deal effectively with FPN over a wide range of temperature, the method presented here provides a good starting point.

\section{ACKNOWLEDGEMENTS}

The authors thank the Natural Sciences and Engineering Research Council of Canada and the Engineering and Physical Sciences Research Council of the UK for their support.

\section{References}

[1] Abbas El Gamal and Helmy Eltoukhy, "CMOS image sensors," IEEE Circuits \& Devices Magazine, vol. 21, no. 3, pp. 6-20, May 2005.

[2] Dileepan Joseph and Steve Collins, "Modeling, Calibration, and Correction of Nonlinear Illumination-Dependent Fixed Pattern Noise in Logarithmic CMOS Image Sensors," IEEE Transactions on Instrumentation and Measurement, vol. 51, no. 5, pp. 996-1001, Oct. 2002.

[3] Gillian F. Marshall and Steve Collins, "A High Dynamic Range Front End for Automatic Image Processing Applications," in Proceedings of the SPIE, May 1998, vol. 3410, pp. 176-85, Advanced Focal Plane Arrays and Electronic Cameras II.

[4] Markus Loose, Karlheinz Meier, and Johannes Schemmel, "A SelfCalibrating Single-Chip CMOS Camera with Logarithmic Response," IEEE Journal of Solid-State Circuits, vol. 36, no. 4, pp. 586-96, Apr. 2001.

[5] Daniel P. Foty, MOSFET Modeling with SPICE: Principles and Practise, Prentice Hall, Upper Saddle River, NJ, 1997.

[6] Dileepan Joseph, Modelling and calibration of logarithmic CMOS image sensors, Ph.D. thesis, University of Oxford, 30 September 2002.

[7] Austria Microsystems, $0.35 \mu \mathrm{m}$ CMOS Process Parameters, Document 9933016.

[8] Austria Microsystems, $0.35 \mu \mathrm{m}$ CMOS Design Rules, Document 9931032.

[9] IMS Chips, "HDRC VGA Imager and Camera Data and Features," Tech. Rep., Institute for Microelectronics Stuttgart, Sept. 2000. 